\title{
THE THEORETICAL INTEGRATIVE MODEL FOR THE LATVIAN CLINICAL PERSONALITY INVENTORY
}

\author{
Jelena Kolesnikova \\ Viktorija Perepjolkina \\ Kristine Martinsone \\ Ainars Stepens \\ Rīga Stradiṇš University
}

\begin{abstract}
The purpose of this article is to present a theoretical integrative model, which reflects contemporary tendencies in the understanding of mental disorders and functional impairment, and which is used as a theoretical frame for the development of the Latvian Clinical Personality Inventory (LCPI). This article, based on the latest research findings in the field, supports the necessity of a combined analysis of mental disorders and functional impairment. Due to scientific findings and deeper understanding of the interrelation between mental disorders and functioning impairment, it has become possible to develop psychological instruments for valid assessment of the disturbances in an individual's cognition, emotion regulation, and behaviour combining with analysis of most essential and relevant aspects of their functioning. An integrative theoretical model of LCPI, developed on the selected criteria from Diagnostic and Statistical Manual of Mental Disorders (DSM - 5; APA, 2013) and WHO International Classification of Functioning, Disability and Health (ICF; WHO, 2001/2015) is presented in the article. Conducted literature analysis permits to conclude, that in a context of clinical personality assessment, a combined analysis of symptoms of mental disorders and relevant functioning criteria is very promising and will be useful in many assessment contexts. Based on such an integrative approach the Latvian Clinical Personality Inventory is currently being developed. This project is a part of the National Research Programme (No. 5.8.2.).
\end{abstract}

Keywords: integrative approach, functioning impairment, Latvian Clinical Personality Inventory, mental disorders, psychological assessment.

\section{Introduction}

On the moment, there is a lack of available clinical personality assessment instruments in Latvia. Some well known and in clinical and research practice widely used personality inventories have been officially adapted in Latvia. For example, one of the most commonly used personality tests in clinical evaluation (Butcher \& Perry, 2008) is Minnesota Multiphasic Personality Inventory - 2 (MMPI - 2, Butcher at al., 1989; Latvian adaptation Sarma, 2005). The Millon Clinical Multiaxial Inventory-III (MCMI-III, Millon et al., 2006) is the other 
one, which is widely used and which was also translated in Latvia recently (Kolesnikova, 2013).

\section{Rationale for the development of the new Clinical Personality Inventory}

The problem with mentioned above assessment instruments is that the copyright holder was authorized the use of these adapted test versions only for specific studies without the right to use them in other studies or for practical purposes. Using such unlicensed test is unethical (ITC, 2001) and means serious administrative penalties because of the breaking of copyrights owned by the copyright enforcement authorities.

The other problem is that these foreign clinical personality inventories are not standardized in Latvia. One more problem is that there is a two-language society in Latvia and local clinical psychologists need parallel versions of one instrument - one version in Latvian language and other - in Russian, because, according to the International Guidelines for Test Use (ITC, 2001), an appropriate test language version must be administered in psychological assessment. However, it is almost impossible to get a permission to adapt mentioned above instruments in Latvia into Russian language, because, in accordance with the official procedure, such an adaptation first of all must be undertaken in Russia, and only then it could be possible to adapt this official Russian version in Latvia.

Therefore, it is clear, that in Latvia up to the moment, there is no clinical personality inventory, which could provide a comprehensive, unbiased and reliable measurement of personality pathology and symptoms of mental disorders. This situation outlines the necessity of developing a clinical personality inventory suitable to the needs and realities of the Latvia's socio cultural context and based on the newest findings in the field. In the frame of the National Research Program (2014-2017; BIOMEDICINE, subproject Nr. 5.8.2.) development of such a clinical personality inventory (Latvian Clinical Personality Inventory; LCPI) was started recently.

The purpose of this article is to present a theoretical integrative model, which reflects contemporary tendencies in the understanding of mental disorders (including personality disorders) and functional impairment, and which is used as a theoretical frame for the development of the Latvian Clinical Personality Inventory (LCPI). 


\section{Theoretical basis for the LCPI clinical scales}

There are two commonly used systems for the classification of mental disorders ${ }^{1}$. One of them currently widely used by practitioners in Europe is The International Classification of Diseases and Related Health Problems (ICD - 10) developed by the World Health Organization (WHO, 1994) ${ }^{2}$. The second classification of mental disorders widely used by practitioners in America and by researches all over the world is the Diagnostic and Statistical Manual of Mental Disorders (DSM). Apparently, its last revision - DSM - 5(APA, 2013) reflects the first (during the last 20 years) major revision of the diagnostic criteria of mental disorders made by DSM-5 work groups integrating into it the latest research findings on mental disorders (APA, 2013).

Much work has been done by scientists at the harmonization of both classifications to achieve the goal of minimizing the differences between these systems (First, 2009; Regier et al., 2013). Nevertheless, some differences still exist. Taking into account, that the DSM-5 reflect the newest findings and tendencies in the defining mental disorders, it was decided to use DSM-5 criteria as a basis of LCPI item development.

In the first stage of the development of the theoretical model of the LCPI individual interviews with Latvian leading clinical psychologists $(N=4)$ and psychiatrists $(N=4)$ was conducted with the aim to find out what clinical scales will be useful and necessary to include in the emerging LCPI. Based on results of these interviews, it was decided to develop following nine clinical scales: Symptoms of Anxiety Disorder, Symptoms of General Depression, Symptoms of Bipolar Disorder, Symptoms of PTSD, Alcohol Problems, Drug Problems, Psychotic Symptoms, Symptoms of Eating Disorders and Somatic Symptoms. In Table 1 information is presented regarding the planed LCPI clinical scales and corresponding mental disorders (based on DSM-5 with ICD-10 codes) which criteria is used for further operationalization on the next stage of scales' item development.

On the next stage of the LCPI development a comparative analysis of the criteria for the selected mental disorders described in DSM-5 (APA, 2013) and

\footnotetext{
${ }^{1} \mathrm{~A}$ mental disorder is a syndrome characterized by clinically significant disturbance in an individual's cognition, emotion regulation, or behavior that reflects a dysfunction in the psychological, biological, or developmental processes underlying mental functioning. Mental disorders are usually associated with significant distress or disability in social, occupational, or other important activities. An expectable or culturally approved response to a common stressor or loss, such as the death of aloved one, is not a mental disorder. Socially deviant behaviour (e.g., political, religious, or sexual) and conflicts that are primarily between the individual and society are not mental disorders unless the deviance or conflict results from a dysfunction in the individual, as described above (APA, 2013, 20). ${ }^{2}$ It should be noted that now there is a revision to ICD-10 and forthcoming ICD - 11 in 2018 (Luciano, 2015).
} 
ICD-10 (WHO, 1992) was conducted with the purpose to select such criteria (within each selected disorder for further operationalization at the item formulation stage) that are more or less similar in both mental disorders classifications. This comparative analysis was necessary to make sure, that the content of the developed items for the each of the planned clinical scales will be corresponding to the selected criteria and that scales score will be interpretable in the frame of both the DSM-5 and ICD-10 codes.

\section{Theoretical basis for the LCPI pathological trait scales}

One part of the LCPI scales are sought to be devoted to a personality pathology. Although personality disorders ${ }^{3}$ (PDs) have been defined categorically throughout the history of psychiatric nomenclatures, researchers have pointed out numerous limitations of this categorical approach (Clark, 2007; Trull \& Durrett, 2005) and suggested that alternative dimensional models provide more validity (Widiger \& Simonsen, 2005). In light of this, the DSM-5 Personality and Personality Disorders Work Group proposed a substantial shift to a dimensional conceptualization and diagnosis of personality pathology (Samuel et al., 2012). In the alternative DSM-5 model for PDs, PDs are characterized by impairments in personality functioning (impairment in ideas and feelings regarding self and interpersonal relationships; self-functioning involves identity and self-direction; interpersonal functioning involves empathy and intimacy [see DSM-5, Section III, APA, 2013, Table 1, p. 762]) and particular constellations of pathological personality traits ${ }^{4}$.

The proposed model comprises 37 maladaptive traits that are said to fall within the six higher-order domains of Negative Emotionality, Introversion, Antagonism, Disinhibition, Compulsivity, and Schizotypy ${ }^{5}$.

\footnotetext{
${ }^{3} \mathrm{~A}$ personality disorder is an enduring pattern of inner experience and behavior that deviates markedly from the expectations of the individual's culture, is pervasive and inflexible, has an onset in adolescence or early adulthood, is stable over time, and leads to distress or impairment (APA, 2013, 645).

${ }^{4} \mathrm{~A}$ personality trait is a tendency to feel, perceive, behave, and think in relatively consistent ways across time and across situations in which the trait may manifest (APA, 2013, 772).

${ }^{5}$ It should be noted that the proposed 37 - trait model was reduced and DSM-5 Section III personality trait system includes five (not six as it was proposed) broad domains of personality trait variation Negative Affectivity (vs. Emotional Stability), Detachment (vs. Extraversion), Antagonism (vs. Agreeableness), Disinhibition (vs. Conscientiousness), and Psychoticism (vs. Lucidity) - comprising 25 specific personality trait facets. These five broad domains are maladaptive variants of the five domains of the extensively validated and replicated personality model known as the "Big Five", or Five Factor Model of personality (FFM), and are also similar to the domains of the Personality Psychopathology Five (PSY-5) (APA, 2013, 773).
} 
Table 1 LCPI Clinical Scales and Corresponding DSM-5 Mental Disorders with ICD-10 codes

\begin{tabular}{|c|c|}
\hline LCPI clinical scales & $\begin{array}{l}\text { DSM-5 mental disorder and ICD-10 }{ }^{\mathrm{a}} \text { codes } \\
\text { in parentheses }\end{array}$ \\
\hline Symptoms of Anxiety & Anxiety Disorders: \\
\hline \multirow[t]{3}{*}{ Disorder } & Generalized Anxiety Disorder (F41.1) \\
\hline & Social Anxiety Disorder (Social Phobia) (F40.10) \\
\hline & Panic Disorder (F41.0) \\
\hline Symptoms of General & Depressive Disorders: \\
\hline Depression & Major Depressive Disorder (F32.0-F32.2) \\
\hline Symptoms of Bipolar & Bipolar and Related Disorders: \\
\hline \multirow[t]{2}{*}{ Disorder } & Bipolar I Disorder (F31.11-F31.13) \\
\hline & Bipolar II Disorder (F31.81) \\
\hline \multirow[t]{2}{*}{ Symptoms of PTSD } & Trauma - and Stressor-Related Disorders: \\
\hline & Posttraumatic Stress Disorder (F43.10) \\
\hline \multirow[t]{2}{*}{ Alcohol Problems } & Substance-Related and Addictive Disorders: \\
\hline & Alcohol Use Disorder (F10.10; F10.20; F10.99) \\
\hline Drug Problems & Unspecified Substance-Related Disorder \\
\hline \multirow[t]{4}{*}{ Psychotic Symptoms } & Schizophrenia Spectrum and Other Psychotic Disorders: \\
\hline & Schizophrenia (F20.9) \\
\hline & Schizophreniform Disorder (F20.81) \\
\hline & Brief Psychotic Disorder (F23) \\
\hline \multirow{4}{*}{$\begin{array}{l}\text { Symptoms of Eating } \\
\text { disorders }\end{array}$} & Feeding and Eating Disorders: \\
\hline & Anorexia Nervosa (F50.0) \\
\hline & Bulimia Nervosa (F50.2) \\
\hline & Binge-Eating Disorder (F50.8) \\
\hline \multirow[t]{3}{*}{ Somatic Symptoms } & Somatic Symptom and Related Disorders: \\
\hline & Somatic Symptom Disorder (F45.1) \\
\hline & Illness Anxiety Disorder (F45.21) \\
\hline
\end{tabular}

Note. ${ }^{\text {a }}$ WHO (1993). ICD-10 Diagnostic Criteria for Research. WHO: Geneva.

It was pointed out that the transition to a dimensional trait model has the potential to addresses several limitations of the previous diagnostic system (Samuel et al., 2012). For example, a dimensional trait system might eliminate the problematic comorbidity across and the heterogeneity within the PDs diagnostic categories by providing a trait profile that is unique to each individual (Widiger \& Trull, 2007). Additionally, such a model holds the promise of improving diagnostic stability as traits have demonstrated greater temporal consistency than diagnostic categories (Morey et al., 2007). The clinical utility of the DSM-5 Section ІП multidimensional personality trait model lies in its ability to focus attention on multiple relevant areas of personality variation in each individual patient (APA, 2013). The DSM-5 Personality and Personality Disorders Work Group also provided a list of traits relevant for describing each of the proposed PDs types (see Samuel et al., 2012; APA, 2013). 
Nevertheless the discussion about the assignments of the pathological personality traits to each of the 10 DSM-5 Section II PDs (e.g. Samuel et al., 2012) are still open, the LCPI work group decided to use this alternative dimensional approach to PDs as a theoretical frame for the development of the LPCI pathological personality trait scales. Based on literature analysis a list of the 40 pathological personality traits - a combination of traits listed in Samuel et al., (2012), Wright et al., (2012) and DSM-5 Section III (APA, 2013) was prepared for further operationalization and developing of the deductively derived 40 LCPI pathological personality trait scales ${ }^{6}$ : Emotional Lability, Histrionism, Anxiousness, Separation Insecurity, Depressivity, Submissiveness, Hostility, Low Self-esteem, Vulnerability, Pessimism, Social Withdrawal, Social Detachment, Intimacy Avoidance, Restricted Affectivity, Anhedonia, Manipulativeness, Deceitfulness, Grandiosity, Will to Power, Attention Seeking, Callousness, Aggression, Anger, Irresponsibility, Impulsivity, Risk Taking/Recklessness, Risk Aversion, Distractibility, Perfectionism, Rigidity, Orderliness, Perseveration, Oppositionality, Unusual Beliefs, Unusual Perceptions, Cognitive Dysregulation, Dissociation Proneness, Eccentricity, Suspiciousness and Self-harm.

Based on the definitions of mentioned above pathological personality traits found in DSM-5 Section III (Table 3, pp. 779-781, APA-2013) and elsewhere including scientific dictionaries a preliminary item pool as operationalization of this traits were prepared. Further steps of the LCPI construction will be psychometrical evaluation of these deductively derived scales and selection of the best performing items for inclusion in the final version of LCPI scales. Additionally, a study to provide an expert consensus description of the DSM-5 ten PDs in terms of the LCPI pathological trait set will be performed. Leading clinical psychologists, psychiatrists and psychotherapists from Latvia will be asked to participate in this research part. This planed research part is necessary because if clinicians and researchers will use these traits to diagnose PD types, it is crucial to be sure that the traits assigned for this purpose are, in fact, relevant to the description of each particular PD.

\section{Theoretical basis for the LCPI functioning scales: The integrative approach for the assessment of mental disorders and functioning impairment}

It is obvious that clinical evaluation of mental health and personality cannot be fully understood, explained or based only on certain narrow criteria. Both

\footnotetext{
${ }^{6}$ It should be noted that trait of guilt/shame we changed for vulnerability, narcissism - for grandiosity, and we have added three more traits - will to power, attention seeking and anger, we believe could clinically relevant.
} 
DSM-5 and ICD-10 does not entail all the outcomes and spectrum about mental disorders, therefore in 2001 World Health organization (WHO) introduced the International Classification of Functioning, Disability and Health ${ }^{7}$, known more commonly as ICF - a classification of health and health-related domains. ICF classifies functioning and disability associated with health conditions, therefore the ICD-10 and ICF are complementary, and users are encouraged to use them together to create a broader and more meaningful picture of the experience of health of individuals and populations (WHO, 2001/2015).

ICF provides conceptual framework to understand and classify functioning based on biopsychosocial approach (WHO, 2001/2015). According to ICF, disabilities (or "functional impairment" in DSM parlance (Ustün \& Kennedy, 2009) is an umbrella term, covering impairments, activity limitations, and participation restrictions. An impairment is a problem in body function or structure; an activity limitation is a difficulty encountered by an individual in executing a task or action; while a participation restriction is a problem experienced by an individual in involvement in life situations. Aspects of health and health-related states summarized under the umbrella term functioning. As the functioning and disability of an individual occurs in a context, ICF also includes a list of environmental factors (WHO, 2001/2015). In accordance to mental disorders the ICF research branch experts have gathered the information provided by numerous studies and reflected it in the ICF Core Sets for schizophrenia, bipolar and depression disorders (ICF Research Branch, 2013 a, b, c).

Functioning is increasingly taken into account for the diagnoses of mental disorders as well as for evaluating the effectiveness of treatments (Cieza et al., 2004; Keeley et al., 2014), especially for the diagnosis and treatment of individuals with major depressive disorder, schizophrenia, bipolar disorders, personality disorders etc. .Various studies approve the association of mental disorders with multiple domains of functioning (e.g. Brutt et al., 2013; Guilera et al., 2012; Guilera et al., 2015; McClure et al., 2013; Hengartner et al., 2014). For example, in the cross-cultural research, based on data derived from the European Study of the Epidemiology of Mental Disorders, a general population study in which adults $(N>21000)$ from Belgium, France, Germany, Italy, the Netherlands and Spain were assessed using the Composite International Diagnostic Interview (mental disorders) and World Health Organization Disability Assessment Schedule second edition (functional disability) was found that mental disorders are related to disability in all domains of functioning:

\footnotetext{
${ }^{7}$ ICF was officially endorsed by all 191 WHO Member States in the Fifty-fourth World Health Assembly on 22 May 2001 (resolution WHA 54.21) as the international standard to describe and measure health and disability.
} 
anxiety disorders the most, followed by mood disorders, and finally alcohol disorders. The findings suggest that mental disorders are associated with similar or higher levels of disability in all ICF domains, except getting around, than arthritis and heart disease (Buist-Bouwman et al., 2006). It was shown that ICF is a helpful conceptual frame for assessment of functional status among people with serious mental illness, promotes a common language in the context of multidisciplinary assessment and integrated treatment model and supports the development of individual rehabilitation plans (Reed et al., 2009).

Taking into account the latest tendencies in the assessment of mental disorders and personality in the context of functioning of individual and empirical evidence of usefulness and importance of such a complex approach, it was decided to use an integrative approach for the development of the LCPI, which will allow to analyse LCPI data not only in a context of symptoms of mental disorders and pathological personality traits, but also in the context of functioning.

To make it possible, an analysis of ICF categories and especially categories included in the Comprehensive ICF Core Set for Depression (Cieza et al., 2004; ICF Research Branch, 2013a), Comprehensive ICF Core Set for Bipolar Disorders (ICF Research Branch, 2013b) and Comprehensive ICF Core Set for Schizophrenia (ICF Research Branch, 2013c) was performed with the aim to select appropriate ICF codes for further operationalization in LCPI items. Based on performed analysis followed by a formal decision-making process a set of 66 ICF categories at the second and third ICF levels with 42 categories from the component body functions (14 second ICF level categories and 28 third ICF level categories) ${ }^{8}, 20$ categories from the component activities and participation (11 second ICF level categories and nine third ICF level categories) ${ }^{9}$, and one ICF first level and four second ICF level categories from the component environmental factors ${ }^{10}$ was prepared. These 66 ICF categories were further operationalized in preliminary version of LCPI items, which were combined in deductively derived functioning scales. Atthe next stage of LCPI development psychometrical analysis of these deductively derived scales will be performed and final versions of LCPI functioning scales will be developed.

\footnotetext{
${ }^{8} \mathrm{ICF}$ categories of the component 'body functions' chosen for the further operationalization in the LCPI items: b126: b1260, b1261, b1262, b1263, b1264, b1265, b1266, b1267; b130: b1300, b1301, b1302, b1303; b1304; b134: b1341, b1342, b1343; b140: b1400; b144; b147: b1470; b152: b1520, b1521, b1522; b156; b160: b1600, b1602, b1603; b164: b1641, b1642; b180: b1800, b1802; b280; b330; b460.

9 ICF categories of the component 'activities and participation' chosen for the further operationalization in the LCPI items: d160; d175; d177; d240: d2400, d2402; d310; d350; d710: d7100, d7102, d7103; d720: d7200, d7202, d7203; d730; d750; d760; d770; d920: d9205.

${ }^{10}$ ICF categories of the component 'environmental factors' chosen for the further operationalization in the LCPI items: Chapter 3: Support and Relationships: e310, e315, e320, e325.
} 


\section{Conclusions}

Latest research findings and tendencies in the psychological assessment reflects the importance and necessity to take into account not only the severity of symptoms of mental disorders but also its consequences, to analyse not only symptoms of a particular disorder, but the whole person in his or her social context. Research findings accept that it is possible to develop such a clinical personality inventory, which would merge mental disorders and functioning into one integrative model.

In the frame of National Research Programme (2014-2017, project No. 5.8.2.) the development of a new multi-item multi-scale self-report measure for the combined assessment of mental disorders and some aspects of biopsychosocial functioning (relevant in the context of personality assessment in clinical and non-clinical settings) was started. LCPI structure includes three parts: clinical scales, pathological personality scales and functioning scales and will provide a possibility to performa combined analysis of mental disorders symptoms and functioning aspects using one clinical personality inventory. Using LCPI specialists will be able to comprehensively evaluate disturbance in an individual's cognition, emotion regulation, and/or behaviour, along with his or her activity limitations, and participation restrictions in result getting extensive and comprehensive individual's psychological profile.

Knowing the severity of symptoms of particular mental disorders, level and aspects of an individual's functioning impairment and his or her pathological trait profile will provide the clinician with a rich base of information and would be valuable in treatment planning and in predicting the course and outcome of many mental disorders in addition to personality disorders. Integrative and complex approach to mental health and personality assessment is very promising and reflects up-to-date tendencies employed in the development of the Latvian Clinical Personality Inventory.

\section{References}

American Psychiatric Association. (2013). Diagnostic and Statistical Manual of Mental Disorders: DSM-5 (5th ed.). Arlington VA: American Psychiatric Association.

Brutt, A. L., Schulz, H., \& Andreas, S. (2013). Development of an ICF-based core set of activities and participation for patients with mental disorders: an approach based upon data. Clinical rehabilitation, 27 (8), pp. 758-767.

Buist-Bouwman, M. A., De Graaf, R., Vollebergh, W. M., Alonso, J., Bruffaerts, R., \& Ormel, J. (2006). Functional disability of mental disorders and comparison with physical disorders: a study among the general population of six European countries. Acta Psychiatrica Scandinavica, 113 (6), 492-500. 
Jelena Kolesnikova, Viktorija Perepjolkina, Kristine Martinsone, Ainars Stepens. The Theoretical Integrative Model for the Latvian Clinical Personality Inventory

Butcher, J. N., Dahlstrom, W. G., Graham, J. R., Tellegen, A., \& Kaemmer, B. (1989). MMPI2: Minnesota Multiphasic Personality Inventory-2: Manual for administration and scoring. Minneapolis: University of Minnesota Press.

Butcher, J. N., \& Perry, J. N. (2008). Personality Assessment in Treatment Planning: Use of the MMPI-2 and BTPI. Oxford: Oxford University Press.

Cieza, A., Chatterji, S., Andersen, C., Cantista, P., Herceg, et al. (2004). ICF Core sets for depression. Journal of Rehabilitation. Medicine, 44, 128-134.

Clark, L. A. (2007). Assessment and diagnosis of personality disorder: Perennial issues and an emerging reconceptualization. Annual Review of Psychology, 58, 227-257.

First, M. B. (2009). Harmonisation of ICD-11 and DSM-V: opportunities and challenges. The British Journal of Psychiatry: The Journal of Mental Science, 195 (5), 382-390. doi: 10.1192/bjp.bp.108.060822

Guilera, G., Gómez-Benito, J., Pino, Ó., Rojo, E., Vieta, E. et al. (2015). Disability in bipolar I disorder: the 36-item World Health Organization Disability Assessment Schedule 2.0. Journal of Affective Disorders, 174, 353-360. doi: 10.1016/j.jad.2014.12.028

Guilera, G., Gómez-Benito, J., Pino, O., Rojo, J. E., Cuesta, M. J. et al. (2012). Utility of the World Health Organization Disability Assessment Schedule II in schizophrenia. Schizophrenia Research, 138 (2-3), 240-247. doi: 10.1016/j.schres.2012.03.031

Hengartner, M., Müller, M., Rodgers, S., Rössler, W., \& Ajdacic-Gross, V. (2014). Occupational functioning and work impairment in association with personality disorder trait-scores. Social Psychiatry \& Psychiatric Epidemiology, 49 (2), 327-335. doi: 10.1007/s00127-013-0739-2

ICF Research Branch. (2013a). Comprehensive ICF Core Set for Depression. https://www.icfresearch-branch.org/download/category/9-mentalhealth

ICF Research Branch. (2013b). Comprehensive ICF Core Set for Bipolar Disorders. https://www.icf-research-branch.org/download/category/9-mentalhealth

ICF Research Branch. (2013c). Comprehensive ICF Core Set for Schizophrenia https://www.icf-research-branch.org/download/category/9-mentalhealth

International Test Commission. (2001). International Guidelines for Test Use. International Journal of Testing, 1 (2), 93-114.

Keeley, J. W., Flanagan, E. H., \& McCluskey, D. L. (2014). Functional impairment and the DSM-5 dimensional system for personality disorder. Journal of Personality Disorders, 28 (5), 657-674. doi: 10.1521/pedi_2014_28_133

Kolvesnikova, J. (2013). Drug-addicted Personality disorders and Problem-solving in the rehabilitation process. Doctoral thesis, University of Latvia.

Luciano, M. (2015). The ICD-11 beta draft is available online. World

Psychiatry: Official Journal of The World Psychiatric Association (WPA), 14 (3), 375-376. doi: 10.1002/wps.20262

McClure, M. M., Harvey, P. D., Bowie, C. R., Iacoviello, B., \& Siever, L. J. (2013). Functional outcomes, functional capacity, and cognitive impairment in schizotypal personality disorder. Schizophrenia Research, 144 (1-3), 146-150. doi: 10.1016/j.schres.2012.12.012

Millon, T., Millon, C., Davis, R., \& Grossman, S. (2006). MCMI-III manual (3rd ed.). Minneapolis: Pearson Assessment.

Reed, G. M., Leonardi, M., Ayuso-Mateos, J. L., Materzanini, A., Castronuovo et al. (2009). Implementing the ICF in a psychiatric rehabilitation setting for People with Serious 
Proceedings of the International Scientific Conference. Volume I, May $27^{\text {th }}-28^{\text {th }}, 2016.388-398$

Mental Illness in the Lombardy region of Italy. Disability \& Rehabilitation, 31, 170173. doi: $10.3109 / 09638280903317955$

Regier, D. A., Kuhl, E. A., \& Kupfer, D. J. (2013). The DSM-5: Classification and criteria changes. World Psychiatry: Official Journal of The World Psychiatric Association (WPA), 12 (2), 92-98. doi: 10.1002/wps.20050

Samuel, D. B., Lynam, D. R., Widiger, T. A., \& Ball, S. A. (2012). An expert consensus approach to relating the proposed DSM-5 types and traits. Department of Psychological Sciences Faculty Publications. Paper 13. http://dx.doi.org/10.1037/a0023787

Sarma, Z. M. (2005). Adaptation of The Minnesota Multiphasic Personality Inventory - 2 to Latvia. Doctoral thesis, University of Latvia.

Trull, T. J., \& Durrett, C. A. (2005). Categorical and dimensional models of personality disorder. Annual Review of Clinical Psychology, Vol. 1, 355-380.

Ustün, B., \& Kennedy, C. (2009). What is "functional impairment"? Disentangling disability from clinical significance. World Psychiatry: Official Journal of The World Psychiatric Association (WPA), 8 (2), 82-85.

Widiger, T. A., \& Simonsen, E. (2005). Alternative dimensional models of personality disorder: Finding a common ground. Journal of Personality Disorders, 19, 110-130.

Widiger, T. A., \& Trull, T. J. (2007). Plate tectonics in the classification of personality disorder: shifting to a dimensional model. American Psychologist, 62, 71-83.

World Health Organization. (1994). International Statistical Classification of Diseases and Related Health Problems, ICD-10, Tenth Revision, Volume 1, 2, 3. Geneva.

World Health Organization. (2001/2015). International Classification of Functioning, Disability and Health (ICF). World Health Organization. Geneva. (Electronical resource) URL: http://www.who.int/classifications/icf/en/

Wright, A. G. C., Thomas, K. M., Hopwood, C. J., Markon, K. E., Pincus, A. L., \& Krueger, R. F. (2012). The hierarchical structure of DSM-5 pathological personality traits. Journal of Abnormal Psychology. 121 (4), 951-95. 